\title{
Analysis of Optics Designs for the LHC IR Upgrade
}

\author{
Tanaji Sen, John Johnstone, FNAL, Batavia, IL 60510
}

\section{Abstract}

We consider the different options proposed for the LHC IR upgrade. The two main categories: quadrupoles first (as in the baseline design) and dipoles-first have complementary strengths. We analyze the potential of the proposed designs by calculating important performance parameters. We also propose a local scheme for correcting the quadratic chromaticity.

\section{INTRODUCTION}

The US-LARP program on the LHC Interaction Region (IR) upgrade is focused on the second phase of the upgrade where the magnets and layout of the IR will be changed and the detectors also upgraded. It is envisioned that the present NbTi magnets in the IR will be replaced by larger aperture magnets built with $\mathrm{Nb}_{3} \mathrm{Sn}$ cable. Several layouts have been proposed for this upgrade including among them a new version of the baseline quadrupole first design and also two flavors of the dipole first design $[1,2]$. The two dipole first designs under study feature in one case triplet focusing with anti-symmetric optics and in the other doublet focusing with symmetric optics in the inner IR magnets. Doublet optics leads to a larger luminosity at the cost of producing elliptical beams at the IP and enhanced chromaticities. Both head-on and long-range beam-beam effects are enhanced with the doublet optics so this option may need dedicated beam-beam compensation in order to offer any advantages. In this report we will analyze in some detail (a) the differences between the quadrupole-first and dipole-first optics with triplet focusing, (b) the advantages of moving the magnets closer to the IP and (c) a local chromaticity correction scheme for the quadrupole-first optics.

\section{COMPARISON OF QUAD-FIRST AND DIPOLE-FIRST}

The requirements on the aperture are about the same in all designs (within 10\%) even though the beta functions are about three times larger in the dipole first designs. The difference arises because both beams are accommodated within a single aperture in the quadrupole-first designs while the beams are separated into different apertures in the dipole-first designs. Figure 1 shows the matched optics through IR5 with $\beta^{*}=0.25 \mathrm{~m}$ for the quadrupole-first and dipole-first optics with triplet focusing. In all cases, extra space has been left for charged particle absorbers TAS and neutral absorbers TAN to cope with the larger particle debris from the interaction point (IP). The first magnets start
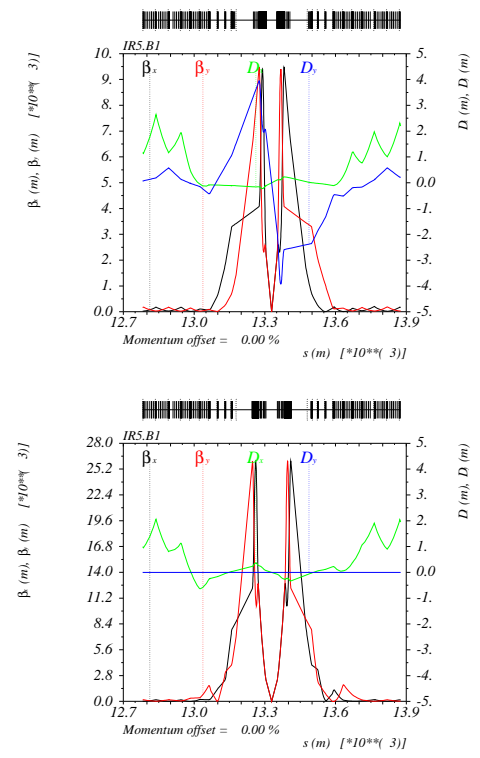

Figure 1: Twiss functions through IR5 for two layouts with $\beta^{*}=0.25 \mathrm{~m}$. Top: quads-first; Bottom: dipoles-first with triplet focusing.

at $23 \mathrm{~m}$ from the IP in both cases but the triplet quadrupoles in the dipole-first case start at $55.5 \mathrm{~m}$ from the IP.

The large $\beta$ functions in the triplets also increase the chromaticity of the ring at collision optics. With the baseline design at $\beta^{*}=0.5 \mathrm{~m}$, the natural linear chromaticity is about -136 units while at $\beta^{*}=0.25 \mathrm{~m}$ this increases to about -200 units. With the dipole-first optics shown above, the chromaticity increases further to about -340 units. This requires larger sextupole strengths for linear and nonlinear chromaticity correction. We will examine this for the quadrupole-first design in a later section.

One of the main advantages of the dipole-first design is the earlier separation of the beams - this reduces the number of long-range beam-beam interactions. The left plot in Figure 2 shows the beam separations (units of $\sigma$ ) against the distance from the IP. The separations for the quadrupole-first case range from 6.9- $12.7 \sigma$ and the interactions extend to $\pm 60 \mathrm{~m}$ from the IP while with the dipolesfirst, the separations are constant at $8.9 \sigma$ and the interactions occur within $\sim \pm 40 \mathrm{~m}$ from the IP. The right plot in this figure shows the tune footprint due to the beam-beam interactions in the two cases. Expectedly, the footprint is smaller in the dipoles-first case especially at amplitudes larger than $2 \sigma$.

The LHC working point is closest to 13 th order resonances but lower order order resonances, 3rd and 10th, are also nearby. We have calculated the resonance driv- 

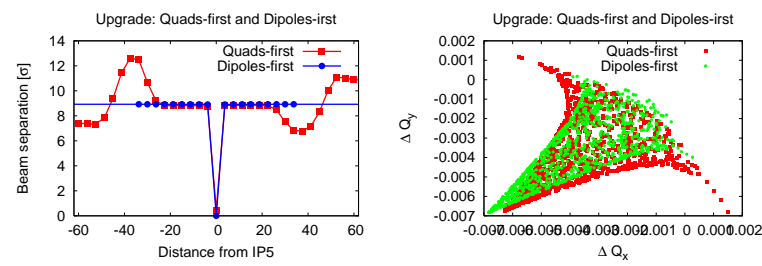

Figure 2: Left: Beam separation at the long-range interactions for the two layouts. Right: Tune footprints up to $6 \sigma$ from beam-beam interactions in the two cases.
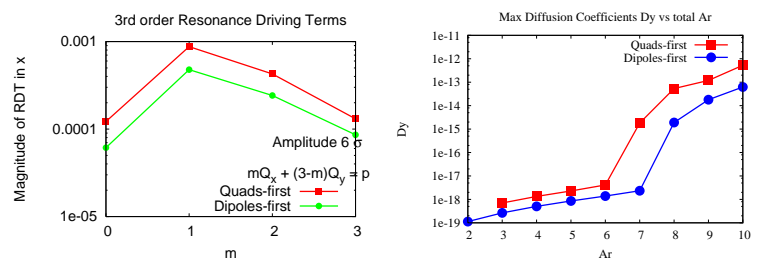

Figure 3: Left: Comparison of 3rd order resonance driving terms; Right: Comparison of a diffusion coefficient vs amplitude for the two layouts

ing terms from the beam-beam interactions using the results in reference [3]. In all cases the resonance strengths are either lower or significantly lower for higher order resonances with dipoles-first. An example is shown of 3rd order resonance terms in the left plot of Figure 3 . The right plot in this figure shows the results of diffusion coefficients from tracking with the code BBSIM. There is a jump in the diffusion at $7 \sigma$ with quads-first, but with dipoles-first the jump occurs at the larger amplitude of $8 \sigma$. Table 1 summarizes the main parameters of the two layouts.

\section{DEPENDENCE ON L*}

It is possible that the IR magnets can be moved closer to the IP in the second phase of the IR upgrade. This would have the benefit of lowering $\beta^{*}$ for the same $\beta^{\max }$ and increasing the luminosity reach. We have examined the relative increase for the two layouts as a function of $\mathrm{L}^{*}$; the

\begin{tabular}{|l|c|c|}
\hline & $\begin{array}{c}\text { Quads } \\
\text { first }\end{array}$ & $\begin{array}{c}\text { Dipoles } \\
\text { first }\end{array}$ \\
\hline$\beta^{\text {max }}[\mathrm{m}]$ & 9484 & 26092 \\
Max aperture [mm] & 101 & 107 \\
Max pole tip field [T] & 10.1 & 10.7 \\
Q' of ring & $-200,-194$ & $-333,-340$ \\
Max 3 ${ }^{\text {rd }}$ order RDT & $0.9 \times 10^{-3}$ & $0.5 \times 10^{-3}$ \\
Max 10 ${ }^{\text {th }}$ order RDT & $0.16 \times 10^{-3}$ & $0.3 \times 10^{-5}$ \\
Beam-beam diffusion & Jump at $7 \sigma$ & Jump at $8 \sigma$ \\
Max ED [mW/g] & -1.0 & 0.6 \\
\hline
\end{tabular}

Table 1: Summary of main parameters for the two IR layouts. Abbreviations: RDT - beam-beam resonance driving term, ED: energy deposition in triplet distance to the first magnet from the IP. For this study the optics was matched to reasonable values at Q4, the first of the outer quadrupoles, as the inner triplet focusing strength increases with lower $\mathrm{L}^{*}$. The luminosity depends directly on $\beta^{*}$ through the spot size at the IP but also indirectly on $\beta^{*}$ via the crossing angle. In order to keep the beam separation constant when $\beta^{*}$ decreases, the crossing angle must be increased as $1 / \sqrt{\beta^{*}}$. This reduces the luminosity gain. If $\mathrm{L}^{*}$ is decreased by more than half the bunch spacing, then the number of long-range interactions also decreases. An empirical scaling relation derived in reference [4] suggests that the crossing angle needs to scale with this number $N_{L R}$ as $\sqrt{N_{L R}}$. It is possible that if some form of longrange beam-beam compensation such as wire compensation were shown to be effective, then the crossing angle would not have to be increased with decreasing $\beta^{*}$. This would allow the full increase in luminosity to be attained. The left plot in Figure 4 shows the increase in luminosity if the crossing angle does indeed have to scale as $\sqrt{N_{L R} / \beta^{*}}$, i.e. without any compensation, for the two layouts. The right plot shows the luminosity gain if the crossing angle stays constant. Long-range compensation together with the quads-first layout could potentially allow a $40 \%$ increase in luminosity if $\mathrm{L}^{*}$ is reduced to $13 \mathrm{~m}$ from the present $23 \mathrm{~m}$. Without an effective compensation scheme, the gains in luminosity are low but instead lower values of $\mathrm{L}^{*}$ may still be useful for gaining operational margin. Instead of lowering $\beta^{*}, \beta^{\text {max }}$ could be lowered while keeping $\beta^{*}$ constant thereby increasing the available aperture in the quadrupoles and lowering the chromaticity.

The two plots in Figure 5 show the change in chromaticity of the insertion as $\mathrm{L}^{*}$ is changed in the two layouts. Figure 6 shows the geometric aberrations due to a multipole error $b_{3}$ in each of the IR quadrupoles for the two layouts. With quadrupoles-first, the aberrations are nearly constant as $\mathrm{L}^{*}$ is reduced below $17 \mathrm{~m}$. On the other hand with the dipoles-first layout, the aberrations are significantly larger and also keep increasing as $\mathrm{L}^{*}$ is reduced. This also shows that the dipoles-first layout will be more sensitive to alignment errors.

\section{NONLINEAR CHROMATICITY CORRECTION}

The correction schemes for the chromaticity and geometric aberrations due to the IR quadrupoles will need to be revisited for the upgrade. We consider here an alternative method for correcting the quadratic chromaticity in the quadrupoles-first layout. The presently envisioned compensating method is via a global scheme using $4 \times 8=32$ sextupole families per beam [5]. The alternative local correction scheme discussed here is based on one proposed in Reference [6] to correct both Q' and Q" with a set of 4 sextupole families per beam. Localized correction has the advantage that the IR's can be operated independently and the global chromaticity and tunes fixed by other constraints.

The local scheme requires using the arc sextupoles in 

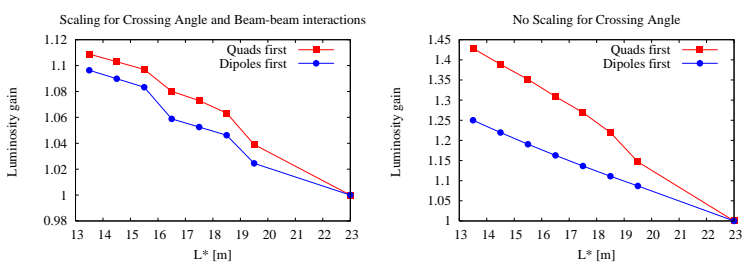

Figure 4: Left: Relative luminosity gain with lower $\mathrm{L}^{*}$ if the crossing angle scales as $\sqrt{N_{L R} / \beta^{*}}$ for the two layouts. Right: The same but in the case that the crossing angle stays constant, for example due to beam-beam compensation.
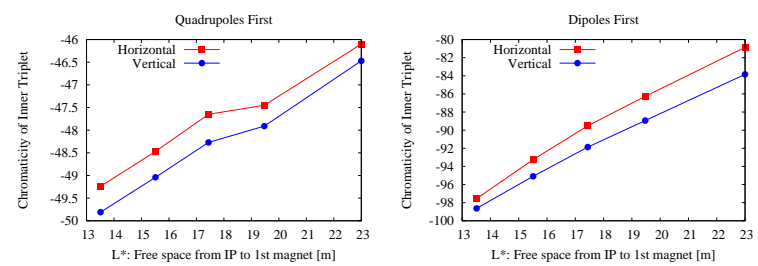

Figure 5: Left: Chromaticity of an insertion with quadsfirst vs L*, Right: The same but for dipole-first.

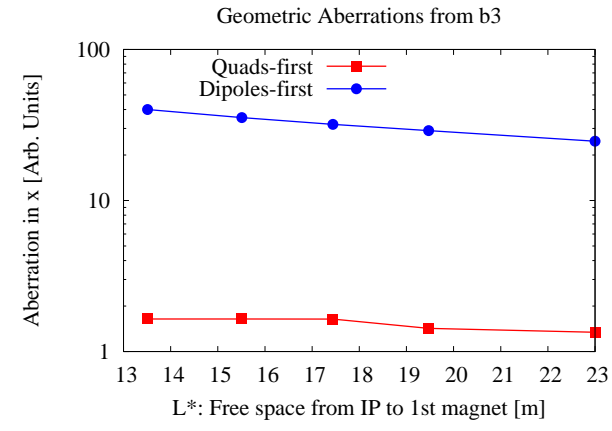

Figure 6: The geometric aberrations of insertions due to the $b_{3}$ multipole in the triplet quads for the two layouts. $b_{3}$ is assumed to be constant.

the 22 cells in each sector bracketing the IR's. With the phase advance in the arc cells close to $90^{\circ}$ in both planes, the fractional tunes across the entire IR plus 44 cell section are tuned to $(.75, .75)$, which helps to reduce the first order chromatic $\beta$-waves. The distribution of sextupoles is illustrated in Figure 7. The SF1 and SD1 families are situated $(2 n+1) \pi / 2$ in phase from the IP. The SF2 and SD2 are interleaved with members of the first families and spaced $m \pi$ in phase from the IP. Correction of the second order chromaticity Q" requires all 4 sextupole families. By adding and subtracting equal strengths to the SF1 and SF2 sextupoles, respectively, Q' is guaranteed not to change to first order, but provides the flexibility to cancel Q" as well. (Similarly for SD1 and SD2). The final sextupole fields are given in Table 2. The maximum available sextupole strength is $4500 \mathrm{~T} / \mathrm{m}^{2}$. Figure 8 shows the tune variation with momentum across the 2 sectors plus IR for $Q^{\prime}=0$, and also both $Q^{\prime}$ and $Q "=0 .\left(\Delta p / p= \pm 3.33 \times 10^{-4}\right.$ is the full bucket size). Correction of the second order terms

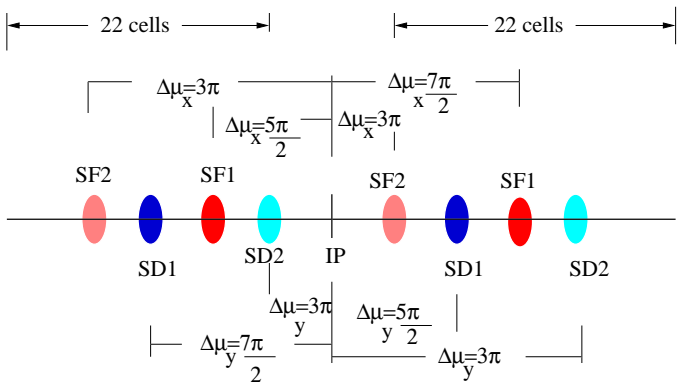

Figure 7: Schematic layout of the sextupoles for 2 nd order chromaticity correction with the quadrupole-first IR optics.

\begin{tabular}{|c|c|}
\hline $\mathrm{SF} 1 / \mathrm{SF} 2\left[\mathrm{~T} / \mathrm{m}^{2}\right]$ & $\mathrm{SD} 1 / \mathrm{SD} 2\left[\mathrm{~T} / \mathrm{m}^{2}\right]$ \\
\hline $2136 / 1955$ & $-4392 /-3984$ \\
\hline
\end{tabular}

Table 2: Sextupole strengths for correcting Q' and Q"

significantly flattens the tune variation. The residual curvature is due to third order terms. Figure 9 shows that after cancelling Q' and Q", $\Delta \beta^{*} / \beta^{*}$ changes are on the level of $1 \%$ across the momentum range

\section{REFERENCES}

[1] Proceedings of the Lumi 05 workshop, Arcidosso, 2005

[2] Proceedings of the Lumi 06 workshop, Valencia, 2006

[3] T. Sen et al, Phys. Rev. ST AB, Vol 7, 041001 (2004)

[4] Y. Papaphilippou and F. Zimmermann, Phys. Rev. ST AB Vol 5, 074001 (2002)

[5] S. Fartoukh, LHC-project-report-308, October (1999)

[6] T. Sen et al, Proceedings of PAC 1993, p 143 (1993)
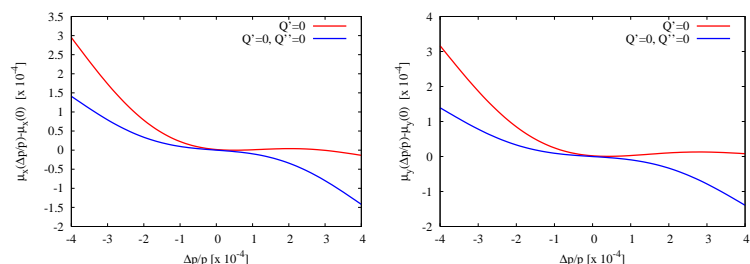

Figure 8: Tune variation (left: horizontal, right: vertical) with only linear chromatic correction and with both linear and quadratic chromatic correction.
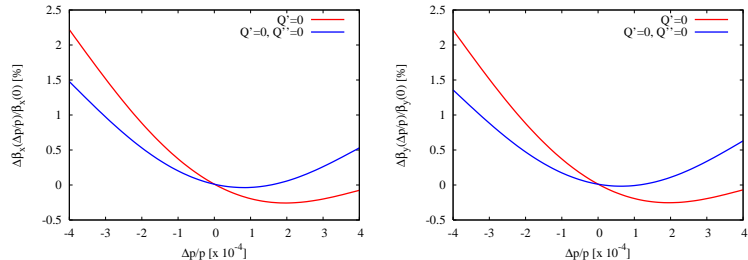

Figure 9: Relative variation in $\beta^{*}$ (left: horizontal, right: vertical) with only linear chromatic correction and with both linear and quadratic chromatic correction. 